\title{
La fertilité humaine, contribution des deux partenaires
}

La conception est un événement aléatoire, le résultat d'une loterie mensuelle, qui fait intervenir deux acteurs : le couple et le temps qui passe... Le couple apporte sa mise par les fertilités de l'homme et de la femme étroitement, quantitativement intriquées, l'hypofertilité d'un des partenaires pouvant être compensée par la bonne fertilité de l'autre. En fertilitéstérilité, l'unité de base est le couple. Les couples totalement stériles sont rares. Le temps, exception faite des couples totalement stériles, apporte à chaque cycle des chances renouvelées. En matière d'infécondité, le temps qui passe est à la fois un critère d'appréciation de la fertilité et un agent thérapeutique de premier ordre...

\section{Daniel Schwartz}

Directeur de l'Unité de Recherches Statistiques de l'INSERM (U2I).

Professeur de Statistiques médicale et biologique à la Faculté de Médecine Paris-Sud

\section{REFERENCES}

I. Leridon H. Human fertility. The basic components. Chicago : The University of Chicago Press, 1977.

2. Schwartz D. Risque et facteurs de risque en épidémiologie et en santé publique. Conc Med 1981 : 745-51.

3. Schwartz D. Importance de la durée d'infécondité dans l'appréciation de la fertilité d'un couple. Population 1 $981 ; 2$ : 237-50.

4. Leridon $H$. Stérilité, hypofertilité et infécondité en France. Population 1982; 4:807-96.

\section{ADRESSE}

D. Schwartz : Inserm (U 2I). 16, av. PaulVaillant-Couturier. 94807 Villejuif Cedex.

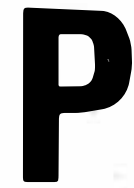

our définir la fertilité, on introduira d'abord la distinction entre fécond : qui a concu (contraire : infécond) et fertile : qui est apte à concevoir (contraire : stérile ou infertile), suivant en cela l'école démographique française [ $\mathrm{I}$ ], dans un domaine où malheureusement la terminologie est cruellement fantaisiste : beaucoup de médecins utilisent indifféremment les deux termes et, pour comble de malchance, les équivalents anglais sont (le plus souvent, mais pas toujours) fertility pour fécondité et fecundity pour fertilité...

La fécondité est un fait, la fertilité une potentialité. Celle-ci est bien plus enrichissante que celle-là, puisque tournée vers l'avenir. Mais bien plus difficile aussi à cerner : comment la définir, cette aptitude? L'indice le plus couramment utilisé est la fécondabilité, qui est la probabilité $P$ de concevoir au cours d'un cycle menstruel. Cette probabilité, pour un couple donné, varie certes d'un cycle à l'autre, avec le rythme des rapports par exemple. Mais on peut admettre [I] qu'elle varie autour d'une valeur moyenne qui caractérise un couple pendant une période donnée. La stérilité correspond à $\mathrm{P}=\mathrm{O}$.
Que la fertilité soit définie par une probabilité, plutôt que par une valeur certaine, comme la taille, n'est pas pour étonner : la conception fait intervenir une série d'étapes : ovulation, fécondation, survie de l'œuf, où triomphe l'aléatoire. Pour un couple de fécondabilité $P$, l'aventure de la procréation est une loterie renouvelée à chaque cycle avec une probabilité $\mathbf{P}$ de gagner.

En bref, la fécondabilité est un risque, tout comme le risque de mort ou d'infarctus ou de mauvaise issue de la grossesse, notion dont on connaît le succès en Santé Publique [2]. Que la conception ne soit pas une maladie ne change rien à l'affaire: elle sera justiciable des techniques classiques de l'épidémiologie.

Deux autres indices sont couramment utilisés. Le premier est basé sur la probabilité de concevoir dans une période plus longue qu'un cycle, telle que 1 ou 2 ans ou $n$ cycles, en fait on considère le complément à 1 , qui est le risque $\mathrm{RI}_{n}$ d'être encore infécond après $n$ cycles. Le second est le délai nécessaire à concevoir (valeur moyenne pour un groupe, ou espérance mathématique pour un couple), soit DNC. Ces deux indices sont bien 
sûr liés à la fécondabilité, il est facile de montrer que, pour un couple :

$$
\mathrm{RI}_{n}=(1-\mathrm{P})^{n} \text { et } \mathrm{DNC}=1 / \mathrm{P} \text {. }
$$

La seconde relation est si simple qu'elle paraît évidente, mais en fait elle demande démonstration. Elle indique que, pour des couples ayant une fécondabilité de, par exemple, $25 \%$, le délai nécessaire à concevoir est, en moyenne, de : $1 / 0,25=4$ cycles.

Il reste à préciser que le mot conception, utilisé dans la définition de la fertilité, demande lui aussi définition. Les démographes considèrent [1] quatre fécondabilités correspondant à quatre définitions de la conception: totale, reconnaissable (conceptions susceptibles d'être reconnues dans l'état des connaissances médicales, ce qui exclut les avortements des premiers jours), apparente (conceptions habituellement reconnues par les femmes elles-mêmes) et effective (conceptions aboutissant à une naissance vivante).

\section{- Mesurer la fertilité}

On ne peut bien sûr pas mesurer pour un couple cette probabilité qu'est la fécondabilité, mais on peut l'estimer par le processus classique en épidémiologie [2]. Ainsi, pour un homme de 50 ans, en France, le risque annuel d'angor, infarctus ou mort subite, est d'environ 4 pour 1000 , tout simplement parce qu'on a dénombré 4 cas sur 1000 sujets. Ce risque, égal pour tous, peut ensuite être diversifié selon le sujet en fonction du niveau pour lui des "facteurs de risque»: s'il a par exemple une tension artérielle systolique de 200, une cholesterolémie de 300, une consommation de 40 cigarettes par jour et un diabète, son risque est de 100 pour 1000 , parce que telle est la fréquence observée (en fait calculée à partir d'un modèle) pour le sous-groupe des sujets présentant ces valeurs [2]. Des estimations analogues, pour la fertilité, quoique moins élaborées, seront indiquées ci-après. Mais sur un point les démographes ont avancé plus loin qu'il n'est habituel en épidémiologie, ils ont pu évaluer dans des groupes, non seulement la fécondabilité, mais sa distribution.

\section{LA FERTILITÉ HUMAINE : VALEUR MOYENNE ET DISTRIBUTION}

Les démographes ont d'abord étudié dans différents pays des séries de couples qui avaient procréé, à une époque où les conceptions prémaritales et la contraception restaient exceptionnelles; ils ont noté le délai nécessaire à concevoir, très variable d'un couple à l'autre, établi la distribution, très étalée, de ces délais. Ils ont été frappés par le fait, apparemment surprenant, que la fécondabilité diminuait de cycle en cycle : si elle était, au premier cycle, de l'ordre de $25 \%$, les couples inféconds au premier cycle n'obtenaient qu'environ $23 \%$ de conceptions au suivant, et ainsi de suite, les couples encore inféconds à 2 ans par exemple n'ayant au cycle suivant qu'une fécondabilité de l'ordre de $8 \%$ [3]. L'hypothèse ingénieuse proposée
[1] fut que la fertilité varie d'un couple à l'autre, de sorte que, les conceptions survenant d'autant plus vite que les couples sont plus fertiles, il ne reste au fil du temps que des couples de moins en moins fertiles.

Partant alors de la distribution observée des délais nécessaires à concevoir, on peut rechercher quelle distribution des fécondabilités l'explique le mieux. Pour les couples jeunes dans nos contrées, la distribution plausible des fécondabilités effectives ainsi déterminée [3] est représentée par la figure 1 , abstraction faite du trait de la classe zéro, puisque tous les couples étudiés avaient procréé. La fécondabilité, autour d'une moyenne $\mathrm{P}=25 \%$, varie très largement de presque zéro

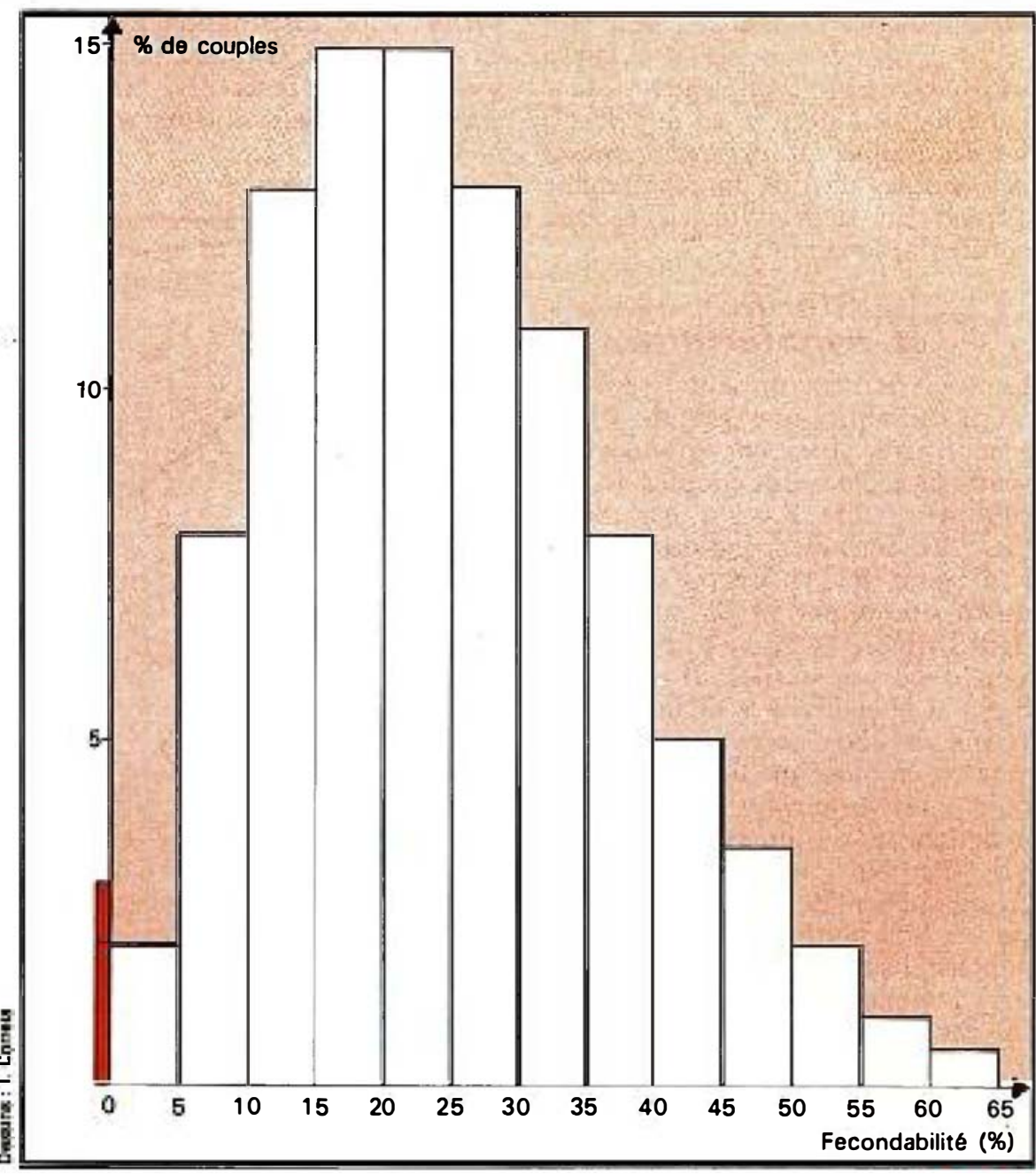

Figure 1. Distribution plausible des fécondabilités (couples jeunes, voir Ref. 3) 
à plus de $50 \%$. Résultat impressionnant, mais non surprenant : les individus sont plus ou moins grands, plus ou moins intelligents; pourquoi les couples ne seraient-ils pas plus ou moins fertiles? D'autant plus qu'ils cumulent les variabilités de deux partenaires...

Les études ci-dessus ne concernaient que les couples féconds. Des enquêtes menées sur la population générale, notamment en France par l'INED [4] ont permis de connaître la proportion de couples ayant cherché sans succès à procréer pendant de nombreuses années et d'estimer ainsi le pourcentage de couples stériles : celui-ci pour les couples jeunes, dans nos contrées, est très faible, beaucoup plus qu'on ne l'admettait classiquement : de l'ordre de $5 \%$, voire $3 \%$. Il est représenté, dans cette dernière hypothèse, par le trait de la classe zéro dans la figure 1 . De cette dernière ressort à l'évidence combien il serait dérisoire, comme on le fait si souvent, de s'enfermer dans l'alternative : stérilité ou fertilité. Il y a des couples stériles, très rares, et des couples fertiles dont la gamme s'étend de la fertilité élevée aux confins de la stérilité.

\section{Stérilité \\ et hypofertilité}

La faible fertilité présente, vis-à-vis de la stérilité, une grande différence, mais aussi une grande ressemblance. Pour des couples de fertilité très faible, $\mathrm{P}=1 \%$ par exemple, les formules indiquées plus haut pour $\mathrm{RI}_{n}$ et $\mathrm{DNC}$ montrent qu'en moyenne $11 \%$ conçoivent dans l'année, $45 \%$ en 5 ans : ils ne sont donc pas stériles. Il reste que $89 \%$ ne conçoivent pas dans l'année et que le délai nécessaire à concevoir est en moyenne de 8 ans : voilà qui, pour beaucoup d'entre eux, évoquera la stérilité et les conduira, un jour ou l'autre, à consulter. Ainsi, la clientèle d'une consultation pour infécondité (trop souvent appelée, hélas, consultation de stérilité) rassemble des couples stériles et des couples de fertilité faible (sans compter quelques uns de bonne fertilité qui n'ont pas eu de chance à la loterie mensuelle ...). Il est important, sur le plan de la Santé Publique, de connaître les poids relatifs des stériles et des hypofertiles dans une consultation pour infécondité.

Partons, à l'instant zéro, d'une population de couples exposés au risque de concevoir et suivons la au fil du temps. Pour des couples de fécondabilité $P$, la proportion de ceux encore inféconds après $n$ cycles; donnée par la formule cidessus $\mathrm{RI}_{n}=(1-\mathrm{P})^{n}$, diminue de cycle en cycle comme indiqué sur la figure 2: après 1 an par exemple (1 2 cycles), presque tous les couples de fertilité moyenne $(\mathrm{P}=25 \%)$ ont conçu, tandis que sont encore inféconds une proportion notable d'hypofertiles $(\mathrm{P}=10 \%)$, la majorité des très hypof ertiles $(\mathrm{P}=1 \%)$ et, bien sûr, la totalité des stériles.

Si la distribution des fécondabilités au départ est celle de la figure 1, l'application de la formule $\mathrm{RI}_{n}=(1-\mathrm{P})^{n}$ donne aussitôt la distribution après $1,2, \ldots, n$ cycles; cette distribution s'enrichit en hypofertiles, d'autant plus qu'ils sont plus hypofertiles, et plus encore en stériles, l'histogramme se tassant progressivement vers la gauche de l'axe, traduisant quantitativement l'observation faite par les démographes, exposée ci-dessus, d'une fertilité moyenne décroissant avec le temps. Or, la distribution des fécondabilités après $n$ cycles n'est autre que celle des couples venant consulter après $n$ cycles d'infécondité; elle représente donc ce que serait le profil d'une consultation pour infécondité, si les couples consultaient tous après $n$ mois d'infécondité. L'allure de cette distribution est résumée, d'après [3], dans 3 hypothèses d'école : celle où les couples viendraient consulter après 6 mois, 1 an ou 5 ans d'infécondité. Le résultat est bien instructif : si les couples attendaient 5 ans pour consulter, sur 100 consultants, 89 en moyenne seraient des couples stériles, les 11 autres seraient fertiles, mais de fertilité très faible. S'ils viennent après 1 an (délai très raisonnable), la majorité $(100 \%-24 \%=76 \%)$ sont fertiles et de fertilité non négligeable $(P=12 \%)$. Mais si les cou-

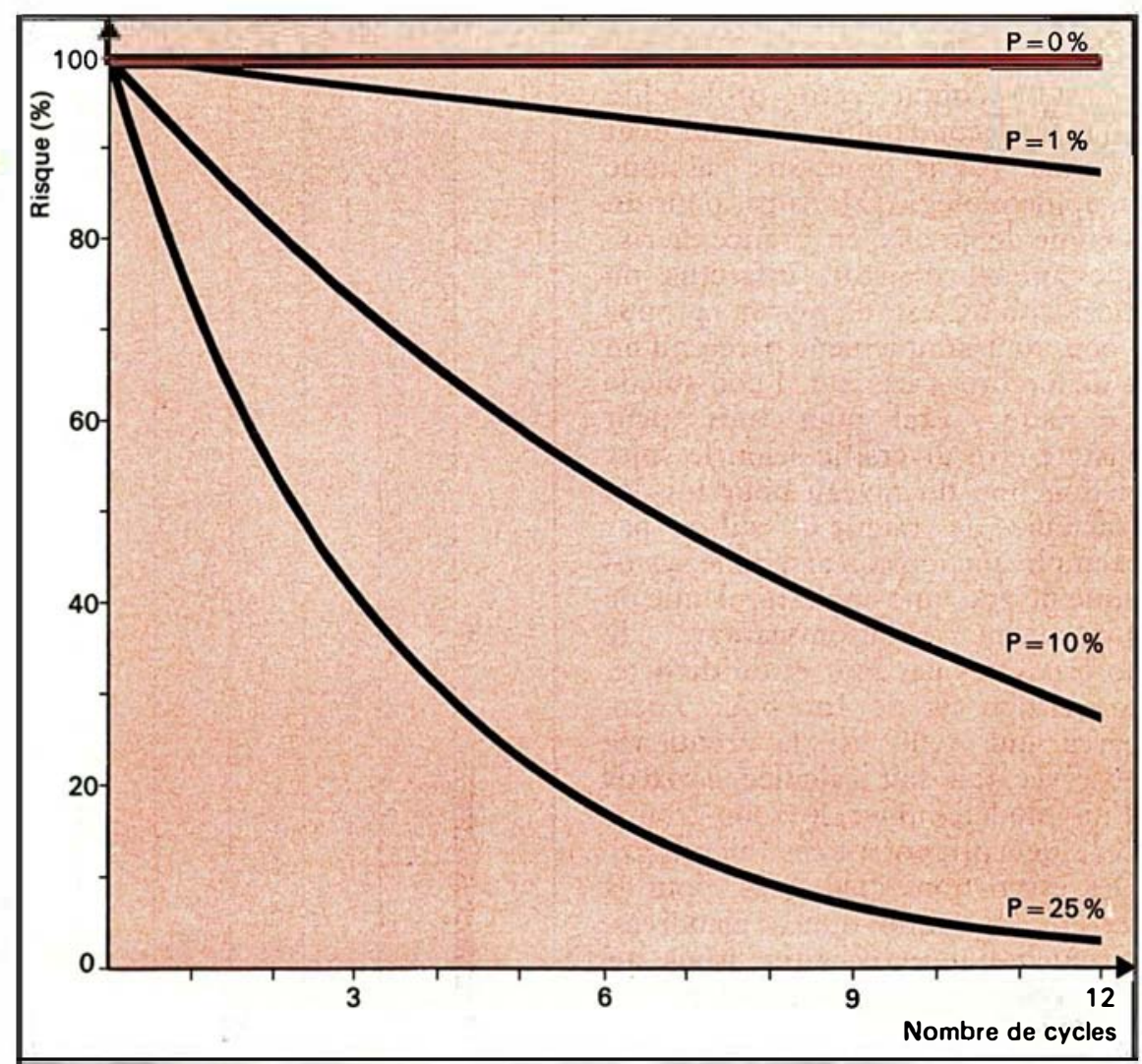

Figure 2 Risque de n'avoir pas confu après $n$ cycles pour des couples de fécondabilités $P=25 \%, 10 \%, 1 \%$ ou $0 \%$ 
ples sont plus impatients, selon la tendance actuelle, et viennent à 6 mois, $89 \%$ d'entre eux sont fertiles et de fertilité relativement bonne $(\mathrm{P}=16 \%)$. Qu'on ne s'étonne donc pas si la majorité des couples consultant actuellement pour infécondité procréent dans les mois qui suivent ...

\section{Durée d'infécondité}

Les résultats ci-dessus peuvent être exprimés autrement. Voici un couple qui vient consulter pour la première fois après 5 ans d'infécondité : il a toutes chances d'être stérile (89 chances sur 100), et s'il est fertile, il l'est très peu $(\mathrm{P}=4 \%)$; de toute manière, fertile ou stérile, si on ne peut faire la distinction, sa fécondabilité présumée est minime (4 pour 1000 ). $\mathrm{Ne}$ pourra-t-on l'assimiler à un couple stérile dans la prise de décision, par exemple l'insémination artificielle si des examens conduisent à incriminer plutôt l'homme? Voici à l'inverse un couple venant consulter à 6 mois : il a de fortes chances d'être fertile, avec une fertilité convenable. D'une manière générale, la connaissance de la durée d'infécondité d'un couple donne une estimation de sa fécondabilité.

Indiquons, à titre d'exemple, que l'information : un an d'infécondité suggère une baisse de fertilité de même ordre que celle d'un examen de sperme concluant à une numération comprise entre o et 10 millions de spermatozoïdes par millilitre, niveau vraiment bas. La durée d'infécondité est donc à elle seule une information de premier ordre dans le bilan exploratoire de la fertilité. Ajoutons qu'elle est simple à obtenir et ne coûte rien.

\section{Le temps,}

\section{moyen thérapeutique}

$\mathrm{Si}$, de plus en plus, les consultations pour infécondité comprennent une majorité de couples non stériles mais hypofertiles, les développements précédents auront suffisamment montré le premier conseil à leur donner : attendre. Pour chiffrer l'importance du facteur temps, indiquons à titre d'exemple que, pour Ioo couples venant consulter à 6 mois, la thérapeutique attendre encore 1 an donnerait en moyenne 64 grossesses! Bien entendu, ce conseil ne saurait être donné aveuglément, sans considération de signes cliniques ou biologiques évidents mais, donné à bon escient, il est efficace, anodin, simple à prescrire ... et bon marché. Le temps est une thérapeutique de premier ordre. S'ensuit une règle trop souvent ignorée : il n'est pas possible d'apprécier un traitement de l'infécondité sans un groupe témoin indiquant, à titre de référence, la fréquence des succès dus au seul écoulement du temps.

\section{FACTEURS DE LA FERTILITÉ HUMAINE}

Si l'on met à part la stérilité due souvent à des causes connues (trompes obturées pour la femme, azoospermie pour l'homme), on peut se demander quels facteurs expliquent, pour les couples fertiles, cette fécondabilité variable de près de zéro à plus de $50 \%$. Par quels moyens les connaitre?

\section{Méthodes}

De la conception, événement à risque, on peut rechercher les facteurs par les méthodes épidémiologiques classiques utilisées pour déceler et chiffrer par exemple le rôle des facteurs d'infarctus. Les principales sont les enquêtes rétrospectives cas-témoins et les enquêtes prospectives [5]. Dans le premier cas, on compare des couples féconds et des couples inféconds pour diverses caractéristiques, notamment les caractéristiques du sperme. Dans le second cas, on suit jusqu'à conception des couples de caractéristiques préalablement évaluées.

On rencontre ici toutefois les difficultés particulières signalées en introduction. La première est que l'unité étudiée est un ensemble de deux personnes. Résultat : quand on compare par exemple le sperme d'hommes féconds et d'hommes inféconds, ces derniers peuvent l'être du fait de leur femme; ce qui diminue la puissance des tests. La deuxième difficulté porte sur le recueil même des données, en raison du secret, voire des tabous entourant la sexualité. Ainsi, dans une enquête prospective entreprise en France sur couples volontaires [6], on a pu obtenir des courbes de température de plus de 4000 femmes, mais d'hommes acceptant de donner du sperme, on n'a pu trouver que ... une dizaine!
Heureusement, pour compenser ces difficultés, la conception se prête à un type d'approche spécifique, grâce au fait qu'elle n'est pas une maladie : on peut "l'inoculer" en quelque sorte d'une manière quasi expérimentale : c'est ce qu'on fait dans l'insémination artificielle avec sperme de donneur (IAD), surtout avec sperme congelé. Ce modèle présente, pour l'explication de la fertilité humaine des avantages multiples et considérables. Le premier, très précieux en raison de la grande variabilité des caractéristiques du sperme d'un éjaculat à l'autre pour un même homme [7], est que le même sperme est connu pour ses caractéristiques et pour sa performance en insémination/succès ou échec. Un autre avantage est la dissociation de fonctions et de facteurs inextricablement liés en reproduction naturelle. Ainsi, la variation de fertilité en fonction d'un facteur aussi simple que l'âge de la femme est difficile à étudier dans les conditions naturelles, parce qu'il n'est guère séparable de l'âge de son partenaire et de la fréquence des rapports, tandis que cette séparation existe d'emblée en IAD. Il n'est bruit actuellement que des problèmes éthiques, juridiques, sociaux, soulevés par de telles dissociations. Mais elles présentent pour la recherche des avantages considérables, dont il serait impardonnable de ne pas profiter. En France, les Centres d'Etude et de Conservation du Sperme (Cecos) sont des banques de sperme à vocation de recherche liées par un protocole commun, situation qui a donné à la France une primauté incontestable pour l'épidémiologie en fertilité humaine. Il faut souhaiter que tous les modes de reproduction artificielle, et notamment la fécondation in vitro, plus 


\section{REFERENCES}

5. Rumcau-Rouquette C., Brcart G., Padieu R Méthodes en épidémiologie. Paris: Flammarion Médecine Sciences, 198 I.

6. Spira A. Enquêtes épidémiologiques sur la fertilité des couples. Génitif 1979; 1 : 29-32.

7. Heuchel V., Schwartz D., Price W. Withinsubject variability and the importance of abstinence period for sperm count, semen volume and pre-freeze and post-thaw motility. Andrologia I98I; $13: 479-85$.

8. Federation Cecos, Schwartz D., Mayaux M. J Female fecundity as a function of age. Results of artificial insemination in 2193 nulliparous women with azoospermic husbands. $N$ Engl 7 Med 1982; 306 : 404-6.

9. Mac Leod J., Gold R. Z. The male factor in fertility and infertility. Fertil Steril 1956; $8: 387-410$.

10. David G., Czyglik F., Mayaux M. J., Schwartz D. The success of A.I.D. and semen characteristics: study on 1489 cycles and 192 ejaculates. Int 7 Androl 1980; 3 :613-9.

II. Jouannet P., Czyglik F., David G. et al Study of a group of 484 fertile men. Part I : distribution of semen characteristics. Int $\mathcal{J}$ Androl 1981; 4 : 440-9.

12. Schwartż D., MacDonald P. D. M., Heuche V. Fecundability, coital frequency and the viability of ova. Popul Stud 1980; $34: 397-400$.

13. Schwartz D., Mayaux M. J., Martin-Boyce A., Czyglik F., David G. Donor insemination conception rate according to cycle day in a series of 821 cycles with a single insemination. Fertil Steril 1979; 2 : 226-9.

14. Schwartz D. Données non publiées.

15. Schwartz D., David D. Données non publiées.

16. Emperaire J. C., Gauzere E., Audebert A Female fertility and donor insemination. Lance 1980; $i$ : 1 423-4.

17. Boué A., Boué J. Évaluation des erreurs chromosomiques au moment de la conception. Biomedicine $1973 ; 18: 372-4$.

18. Boué J., Boué A. Anomalies chromosomiques dans les avortements spontanés. Les accidents chromosomiques de la reproduction. In : comple rendu du Collogue Inserm-Dgrst-Oms 1973 : 29-55.

\section{TIRES A PART}

D. Schwartz : Inserm (U 21). 16, av. PaulVaillant-Couturier. 94807 Villejuif Cedex.

riche encore que l'IAD de possibilités expérimentales fassent profiter la science des avantages de l'artifice.

Des divers facteurs étudiés, dont le rôle sur la fertilité a été chiffré, on ne citera que quelques uns.

Des facteurs féminins, on ne retiendra que l'âge de la femme, que l'IAD a permis d'étudier en l'isolant des facteurs d'habitude associés. Une récente enquête, portant sur plus de 2000 femmes [8] a permis de déceler une baisse de fertilité, faible mais significative, dès l'âge de 30 ans. Résultat qui a provoqué aux Etats-Unis un violent débat sur un choix essentiel : les femmes doivent-elles procréer d'abord et travailler ensuite, plutôt que l'inverse? Débat disproportionné avec le résultat publié, du fait d'une mauvaise interprétation : d'abord l'augmentation du taux de femmes infécondes à 1 an qui était signalé a été assimilé à tort à une augmentation du taux de femmes devenues stériles, ensuite la baisse de fertilité est en tout état de cause très faible.

Des facteurs masculins, on retiendra surtout qu'ils existent, bousculant là encore une idée profondément ancrée, celle de la responsabilité uniquement féminine - que traduit d'ailleurs le terme passif de fécondabilité. On a mis en évidence une si étroite relation entre la fertilité et certaines caractéristiques du sperme concentration, mobilité, morphologie des spermatozoïdes $[9,10]-$ et celles-ci sont si variables d'un homme à l'autre, ceci dans une population d'hommes fertiles [11], qu'on comprend déjà pour une bonne part la variabilité de la fertilité des couples.

En fait, la responsabilité est partagée. D'abord par l'intermédiaire du rythme des rapports. Bien qu'es- sentiel, ce facteur est mal connu, en raison du manque de données. On considère comme plausibles les ordres de grandeurs découlant de données extrapolées par un modèle mathématique [12]: si on désigne par $i$ l'intervalle entre les rapports (rapports tous les $i$ jours) et par $P$ la fécondabilité, on a :

\begin{tabular}{|c|c|c|c|}
\hline $\begin{array}{l}P=7 \\
P=16 \%\end{array}$ & $22 \%$ & $31^{3} \%$ & $\frac{1}{49 \%}$ \\
\hline
\end{tabular}

Bicn entendu, les rapports n'interviennent pas seulement par leur fréquence, mais par leur disposition dans le cycle. Les jours les plus fertiles sont ceux qui précèdent immédiatement le jour "zéro ", dernier point bas de la courbe thermique [13]. Signalons à ce sujet que des couples essaient de "viser" au mieux pour choisir le moment de la conception, souvent en respectant une abstinence suivie d'un rapport aussi près que possible du jour zéro : or, non seulement le jour zéro n'est probablement pas le jour de fécondabilité maximum, mais en outre à le viser on risque de tomber trop tard. Aussi, les conséquences sont-elles très ... écologiques : la conception chez ces couples est retardée d'environ 2 mois ... [14]. La responsabilité partagée provient aussi et surtout d'une étroite conjugaison des caractéristiques masculines et féminines. On ne saurait mieux illustrer cette conjugaison que par l'exemple ci-après, extrait des données d'IAD du CecosBicêtre [1 5], qui indique (tabl. I) la fécondabilité en fonction d'un facteur féminin, la qualité de la glaire cervicale, et d'un facteur masculin, le taux de spermatozoïdes mobiles dans le sperme qui a subi la congélation. On voit quels rôles jouent, et la qualité de la glaire (dans chaque colonne) et celle du sperme (dans

Tableau I

TAUX DE SUCCÉS PAR CYCLE EN I.A.D. SELON LA QUALITÉ DE LA GLAIRE ET LE TAUX DE SPERMATOZOÏDES MOBILES APRĖS DÉCONGÉLATION

(effectif total $=6327$ cycles d'insémination, voir Ref. 15)

\begin{tabular}{|c|c|c|c|c|}
\hline pourcentage de mobiles (\%) & $\leqq 40$ & 45,50 & 55,60 & $>60$ \\
\hline glaire peu abondante & $5 \%$ & $8 \%$ & $9 \%$ & $14 \%$ \\
\hline glaire abondante & $10 \%$ & $11 \%$ & $17 \%$ & $20 \%$ \\
\hline
\end{tabular}

$m / s$ mars 85 
chaque ligne), dans une conjugaison spectaculaire puisque le taux de succès varie de $5 \%$, quand les deux sont mauvaises, à $20 \%$ quand les deux sont bonnes.

Un autre exemple est la comparaison, dans l'étude [16], des taux de succès obtenus selon que le mari de la femme inséminée a un sperme sans spermatozoïdes (azoosperme) ou pauvre en spermatozoïdes (oligozoosperme): le taux est significativement plus bas pour l'oligozoospermie $(5 \%)$ que pour l'azoospermie $(12 \%)$. Ce résultat paraît surprenant : pourquoi intervient en IAD le sperme du mari? Et dans un sens curieux : plus il est mauvais, meilleur est le résultat? L'interprétation la plus plausible est la suivante : un homme oligozoosperme n'est pas stérile, mais hypofertile, il a ses chances, d'autant plus grandes que sa femme est plus fertile. S'il en arrive à l'IAD après plusieurs années d'insuccès, c'est que sa femme sans doute n'est pas très fertile, le taux de succès en.IAD faible est donc dû à sa femme.

En définitive, dans la loterie mensuelle, l'homme et la femme apportent chacun leur mise, faible ou forte; elles se combinent pour le meilleur et pour le pire. Ainsi donc que celui-là ne s'étonne pas qui, venu consulter pour infécondité, et dont le sperme s'avère médiocre, s'entend dire qu'il faut traiter sa femme ... En fertilité-stérilité, l'unité de base est le couple.

\section{ANALYSE DE LA FÉCONDABILITÉ}

A mesure que progressent les recherches, on connaît un nombre croissant de facteurs de la fécondabilité, facteurs de la femme, de l'homme, du couple, les uns permanents ou durables, les autres particuliers à un moment (caractéristiques propres d'un cycle ou d'un éjaculat). Pour avoir une vue plus claire des déterminants de la fertilité, il est nécessaire de savoir à quelle étape de la conception ils interviennent.

La fécondabilité $P$ peut être exprimée selon la formule :

$$
\mathrm{P}=\mathrm{P}_{0} \times \mathrm{P}_{s} \times \mathrm{P}_{v}
$$

par le produit des probabilités que soit émis un ovule $\left(\mathrm{P}_{0}\right)$, qu'il soit fécondé $\left(P_{f}\right)$ et que l'œuf formé soit viable $\left(\mathrm{P}_{v}\right)$. La viabilité de l'œuf traduite par $\mathbf{P}_{v}$ peut concerner une durée plus ou moins longue, depuis un très court instant jusqu'à la durée totale de la grossesse, ce qui reflète l'ambiguïté du mot conception, et ramène aux différentes fécondabilités envisagées plus haut : totale, reconnaissable, apparente, effective.

Un fait extrêmement frappant est que les facteurs de fécondabilité actuellement connus, mesurés, étudiés, concernent essentiellement $\mathrm{P}_{0}$ (explorations gynécologiques visant à évaluer la qualité de l'ovulation) ou $P_{f}$ (examens de sperme portant sur la numération, la mobilité, etc.) mais pas $\mathrm{P}_{v}$. Or cette probabilité joue un rôle majeur, car on admet que la mortalité embryonnaire est de l'ordre de $50 \%$ [17], entraînant à elle seule une réduction de moitié de la fécondabilité. Sans doute sait-on que cette mortalité est le fait d'aberrations chromosomiques dans la majorité des cas [18], mais l'étiologie de ces aberrations est mal connue. On a souvent évoqué le rôle d'un vieillissement des gamètes par attente au moment de la fécondation, rôle abondamment prouvé chez les animaux, et qui pourrait être particulièrement important dans l'espèce humaine, du fait qu'elle se singularise par l'absence de synchronisation dans l'émission des gamètes mâle et femelle. Mais précisément parce que l'espèce humaine est singulière, les résultats observés chez l'animal peuvent-ils être extrapolés?

Ainsi, l'étiologie de la mortalité embryonnaire précoce est à la fois d'un haut intérêt sur le plan biologique, et d'un abord quasi impossible en reproduction naturelle puisqu'il s'agit d'avortements trop précoces pour être décelés chez la femme, et que l'acquis sur l'animal suscite des réserves. Ici encore, les modes de reproduction artificielle et, singulièrement, la fécondation in vitro devraient constituer des approches privilégiées

\section{Summary}

Conception is a random event, the result of a monthly lottery which includes two actors: the couple and the course of time... The couple places its bets on their respective fecundities, which are tightly - and quantitatively - intertwined, the subfecundity of one being balanced by the fecundity of the other. In the study of fecundityunfecundity, the couple is the basic unit of study. It is rare that a couple is totally sterile. But fecundity varies considerably from one fecund couple to another, and clinics, wrongly designated "for sterility", when they take care, in fact, of subfecund couples.

Time, except in the case of truly sterile couples, brings renewed chances for success with each cycle. In cases of unsuccessful attempts at pregnancy, the course of time is both a measure of the level of fecundity, and an outstanding therapeutic agent ... In the evaluation of fecundity, the determination of factors is the result of both demographic and epidemiological studies. The element of risk surrounding conception is amenable to the same epidemiological techniques as those established for the risk of illness. Yet their application is more difficult here than in any other domain, both because of the privacy surrounding sexuality, and because the basic unit involved is composed of two people. In compensation, experimental approaches are possible, which would not be in the case of illness: those offered by artificial reproduction. 